\title{
La noción de cuerpo en los escritos maduros de Leibniz
}

\author{
[Leibniz's Account of Body in His Mature Writings]
}

\author{
RODOLFO EMILIO FAZIO \\ Universidad de Buenos Aires \\ Consejo Nacional de Investigaciones Científicas y Técnicas \\ rodolfofazio@gmail.com
}

Resumen: En el presente trabajo analizo el concepto de cuerpo en los escritos maduros de Leibniz. En el marco del debate contemporáneo acerca del estatus de la sustancia corpórea examino el entramado de las tres nociones sobre las que se construye la metafísica leibniziana de los cuerpos, a saber, la de materia prima, la de cuerpo orgánico y la de extensión. En cada caso evalúo el estatus ontológico que Leibniz les reconoce así como el papel que cumplen en su metafísica.

Palabras clave: metafísica, materia prima, materia segunda, cuerpo orgánico, extensión

\begin{abstract}
In this paper I examine the concept of body in Leibniz's mature writings. In the context of the contemporary debate on the status of the corporeal substance, I analyze the relation between the three notions from which he builds his metaphysics of bodies, namely, the primary matter, the organic body, and the extension. For each case I evaluate their ontological status and the role they play in his metaphysics.
\end{abstract}

Key words: Metaphysics, primary matter, secondary matter, organic body, extension

\begin{abstract}
Recordarás que, según mi opinión, no sólo todas las vidas, todas las almas, todas las mentes, todas las entelequias primitivas son perennes, sino también que cada entelequia primitiva o cada principio vital tiene unida perpetuamente una máquina de la naturaleza que recibe el nombre de cuerpo orgánico; aunque esta máquina conserve brevemente su figura, ella consiste en el flujo y se repara de manera perpetua, como la nave de Teseo.
\end{abstract}

Carta de Leibniz a Wagner ${ }^{1}$

Las bases de la metafísica madura de Leibniz y, en particular, de las nociones de cuerpo y sustancia corpórea que figuran en ella han despertado un interés renovado entre los exégetas contemporáneos. En

${ }^{1}$ GP VII, 530 (a menos que se indique lo contrario, las traducciones son mías).

Diánoia, volumen LXIII, número 80 (mayo de 2018): pp. 29-52. 
particular, en los últimos años Phemister 2005, Arthur y Loptson 2006 o Hartz 2007 han propuesto interpretaciones que, alejándose de las lecturas idealistas tradicionales, buscan explicar cómo, incluso en el sistema monadológico, puede hablarse de sustancias corpóreas genuinas. ${ }^{2}$ Más allá de sus aciertos o inconvenientes, estas propuestas hermenéuticas, calificadas usualmente de realistas, constituyen una contribución significativa a la investigación leibniziana porque revelan la complejidad de la posición madura del filósofo alemán, quien, lejos de negarle estatus ontológico al cuerpo, reconoce hasta el final de su vida no sólo que hay sustancias corpóreas, sino incluso que toda sustancia finita es de esa clase. Con el fin de ofrecer una aportación al debate acerca del estatus de la sustancia corpórea en la filosofía leibniziana de madurez, analizaré uno de los conceptos que figuran en ella: el de "cuerpo". Para ello divido mi trabajo en tres secciones. En primer lugar, examinaré la noción leibniziana de "materia prima", precisaré sus particularidades frente a las posiciones tanto tradicionales como modernas y evaluaré su papel en la constitución de la mónada. En segundo lugar, investigaré qué entiende Leibniz por "cuerpo orgánico" y especificaré sus principales características. En tercer lugar, consideraré el vínculo que estas nociones guardan con la "extensión". Si bien no entraré en el debate acerca de la sustancia corpórea, comprender el entramado que Leibniz teje entre la materia prima, la materia segunda y la extensión permite sentar bases más sólidas para esa discusión.

\section{La materia prima de la mónada}

Desde sus primeras publicaciones sobre metafísica, en 1694 y 1695 , hasta sus últimos escritos y debates epistolares, Leibniz cuestiona uno de los pilares de la filosofía cartesiana, a saber, la res extensa. En especial, el filósofo alemán rechaza que la "extensión" pueda constituir una propiedad esencial de una cosa y, en lugar de ella, introduce la noción de "materia prima". A pesar de su filiación terminológica con la escolástica, Leibniz comprende este concepto de un modo particular. En esta sección expondré las características generales de la noción leibniziana de "materia prima", analizaré su papel en la constitución de las sustancias finitas y evaluaré su estatus ontológico.

Si bien la noción de materia prima puede rastrearse en todo el pensamiento de Leibniz, aparece por primera vez para el gran público en

\footnotetext{
${ }^{2}$ Para un resumen del debate entre las diversas posiciones sobre la sustancia corpórea, cfr. Hartz 2008 y Rutherford 2008.
}

Diánoia, vol. LXIII, no. 80 (mayo de 2018). 
el Specimen dynamicum (1695). ${ }^{3}$ En este ensayo, Leibniz ofrece una caracterización de los principales elementos que estructuran su metafísica de madurez. Respecto del concepto que nos ocupa, afirma lo siguiente:

La fuerza primitiva de padecer o resistir constituye lo mismo que, si se ha interpretado de manera correcta, se denomina en las Escuelas materia pri$m a$, gracias a la cual evidentemente logra que el cuerpo no sea penetrado por otro cuerpo y se opone una resistencia y, al mismo tiempo, está dotado de cierta inercia natural, por así decir, esto es, de una repugnancia al movimiento, y no soporte por ello ser impulsado por la fuerza del agente a no ser que quebrante algo en éste. (GM VI, 236-237)

Leibniz equipara la materia prima con un concepto de cuño personal: la "fuerza primitiva pasiva". En principio, pueden destacarse dos notas generales de esta noción. Por una parte, se concibe como la capacidad que tienen las cosas para obstaculizar una acción, esto es, el paso de un estado o predicado a otro. Cabe subrayar que no se trata de la mera impenetrabilidad que diversos filósofos modernos, y el propio Leibniz en su juventud, habían reconocido como una característica esencial de la materia, esto es, como la capacidad de ocupar un lugar e impedir que otra cosa lo ocupe, sino que en verdad se piensa como una resistencia activa. Esta concepción de la pasividad se encuentra ya en textos de inicios del periodo medio como en Definitiones cogitationesque metaphysicae (1678), donde Leibniz sostiene que "resistente es lo que actúa en eso por lo que padece" ( $A$ VI, 4, 1394), y también en el mismo Specimen dynamicum, en el cual sostiene que "la pasión de todo cuerpo es espontánea" (GM VI, 251). ${ }^{4}$ De este modo, la fuerza pasiva constituye un impedimento para superar al cual se requiere un esfuerzo del agente. Por otra parte, esta potencia se piensa como algo invariante que subyace en los distintos cambios en cada una de las cosas: en ello radica su carácter primitivo. A diferencia de la fuerza derivativa propia de la

${ }^{3}$ Aun cuando Leibniz recurre a la materia prima en su metafísica tanto juvenil como madura, este concepto sufre variaciones importantes a lo largo de su pensamiento. En líneas generales pueden distinguirse dos momentos en su desarrollo. Entre 1663 y 1677 se concibe como algo extenso e impenetrable que llena el espacio, que está dividida actualmente al infinito y que, indeterminada por sí, requiere del movimiento para determinarse (cfr. A VI, 2, 279-280). Desde 1678 Leibniz se aparta de su primera concepción y equipara la materia prima con la fuerza primitiva pasiva. En mi trabajo me concentro sólo en esta segunda noción, que es la que se presenta y discute en sus escritos maduros.

${ }^{4}$ Sobre la concepción de la materia y la sustancia corpórea en el periodo medio, cfr. Fazio 2017. 
dinámica leibniziana, que es momentánea y varía constantemente en los cuerpos, la potencia primitiva es una noción propia de la metafísica y es una fuerza constante y propia de cada sustancia que subyace en sus múltiples cambios. De este modo, en la metafísica leibniziana la fuerza primitiva pasiva no es sino el freno interno e invariante que una sustancia tiene frente a su propia espontaneidad, esto es, frente a su capacidad de producir los cambios por sí sola si no hay nada que se lo impida. ${ }^{5}$ Si bien en el Specimen dynamicum se habla de la materia prima en relación con los cuerpos, se trata de un elemento que, como veremos a continuación, forma parte de toda sustancia finita.

La materia prima ocupa un lugar entre los elementos más básicos de la metafísica leibniziana. De hecho, cumple un papel protagónico en la constitución de las sustancias finitas. Ello se evidencia en los intercambios epistolares con De Volder y Des Bosses:

Distingo, por lo tanto, lo siguiente: [1] la entelequia primitiva o alma; [2] la materia prima o potencia pasiva primitiva; [3] la mónada completa formada por estas dos; [4] la masa o materia segunda, esto es, la máquina orgánica, a la que concurren innumerables mónadas subordinadas; [5] el animal o sustancia corpórea, a la que la mónada dominante da unidad dentro de la máquina. (GP II, 252; trad. OFC XVI, 1200)

La materia primera es esencial para cualquier entelequia y no se separa nunca de ella, ya que le da completitud y es la misma potencia pasiva de toda la sustancia completa [...]. Entonces, aunque Dios pueda por potencia absoluta privar de materia segunda a una sustancia creada, no la puede privar de materia primera, ya que entonces haría un acto puro que solo él mismo es. (GP II, 324; trad. OFC XIV, 199-200)

La potencia pasiva primitiva es aquello que limita a la fuerza primitiva activa o forma sustancial en cada una de las cosas de un modo particular. $^{6}$ Como puede observarse en el segundo pasaje, la materia prima es lo que distingue a las sustancias finitas de la sustancia infinita y, por causa de ello, ni siquiera Dios mismo se las puede quitar. Ahora bien, respecto de su papel en la constitución de las mónadas, Leibniz advierte que la materia prima no es por sí sola ni una sustancia ni un fenómeno, pues sin una forma sustancial o fuerza primitiva activa no es más que algo incompleto. Tal como le comunica a Bernoulli ya en 1698:

${ }^{5}$ Para una presentación general de la noción de fuerza primitiva pasiva, $c f r$. Garber 2009, pp. 122-123.

${ }^{6}$ Sobre la caracterización general de la sustancia en términos de fuerza primitiva activa, cfr. Fazio 2016b.

Diánoia, vol. LXIII, no. 80 (mayo de 2018). 
"La materia misma por sí sola, esto es, como mole, a la que puedes llamar materia prima, no es sustancia ni tampoco es agregado de sustancias, sino algo incompleto" (GM III, 537). Esta tesis persiste incluso en los textos posteriores a la Monadologie. Por ejemplo, en un carta a Remond de 1715 sostiene que "la pura materia prima tomada sin las almas o vidas a las que está unida es enteramente pasiva: también, si se habla con propiedad, no es una sustancia, sino algo incompleto" (GP III, 657). ${ }^{7}$ Con ello, Leibniz enfatiza que la materia prima y la forma sustancial son en verdad dos elementos que se necesitan entre sí para la constitución de la sustancia finita o mónada completa. Ahora bien, tal como indica Phemister 2005 (p. 40), la cuestión radica en comprender en forma adecuada qué quiere decir Leibniz con "incompleto", pues no basta con afirmar que "lo incompleto es lo activo sin lo pasivo y lo pasivo sin lo activo" (GM III, 551). En efecto, esta presentación general podría hacernos pensar que Leibniz retoma la teoría escolástica de las sustancias completas e incompletas. Para mostrar la diferencia entre la concepción leibniziana de la materia prima y la propia de la escolástica, es necesario precisar cómo comprende Leibniz el carácter incompleto de ella.

Así, un primer interrogante sobre la noción leibniziana de materia prima es el siguiente: ¿qué es la materia prima sin la forma sustancial? O, en las palabras dirigidas a Bernoulli, ¿qué es lo pasivo sin lo activo? Una respuesta posible y frecuente es la que en los últimos años ha desarrollado en detalle Pauline Phemister, quien plantea que, al ser algo puramente pasivo, la materia prima por sí sola sólo puede existir como potencialidad. ${ }^{8}$ En defensa de su lectura, la autora recuerda que tanto en De ipsa natura (1698) como en los Nouveaux essais (1704) Leibniz afirma que la materia prima es algo puramente pasivo y, por lo tanto, incompleto (cfr. GP IV, 512; A VI, 6, 378). Sin embargo, estas referencias no ayudan a determinar la respuesta al interrogante inicial, pues sólo reiteran lo que Leibniz le dijo a Bernoulli: que algo pasivo, esto es, sin actividad, es incompleto. Entonces, la cuestión es determinar si es lícito concluir que la materia prima, sin forma sustancial, existe como potencialidad. No obstante, ipuede afirmarse en la metafísica leibniziana de madurez que la materia prima es un sustrato que existe potencialmente a la espera de una entelequia que lo complete? Pienso que no, porque esta comprensión de la materia prima no logra explicar un punto fundamental de la posición de Leibniz. En efecto, afirmar que la materia subsiste en potencia y se actualiza mediante una forma es

\footnotetext{
${ }^{7}$ Para otros pasajes similares, cfr. GP II, 206 y 378.

${ }^{8}$ Phemister sostiene que la materia prima "al ser puramente pasiva, sólo puede existir como potencialidad" (Phemister 2005, p. 44).
} 
hacer una lectura en clave escolástica, esto es, pensar la materia prima como una sustancia incompleta. Esta interpretación podría ser adecuada para los escritos juveniles de Leibniz, pero no refleja su posición madura. ${ }^{9}$ Con el fin de entender por qué esta exégesis es inadecuada basta recordar que la materia prima se define justo como potencia primitiva pasiva. Por ello, cuando Leibniz afirma que la potencia primitiva pasiva no existe sin forma sustancial - esto es, sin potencia primitiva activa-, no se dice que exista como algo potencial, sino todo lo contrario, que no existe ni siquiera como algo potencial, pues no existe la potencia pasiva sin la activa. De este modo, la materia prima no es un sustrato pasivo sobre el cual actúa la forma sustancial, sino que, tal como Leibniz precisa a De Volder: "Dicho con propiedad y rigor, tal vez no deberíamos decir que la entelequia primitiva actúa sobre la masa de su cuerpo, sino que se junta con la potencia pasiva primitiva a la que completa y con la que constituye la mónada" (GP II, 250; trad. OFC XVI, 1199).

En este punto Leibniz invierte la tesis escolástica y sostiene que la materia prima no tiene prioridad ni ontológica ni lógica respecto de la mónada, la cual se erige como fundamento primero en ambos órdenes. De este modo, la potencia primitiva de resistencia sólo subsiste sin la forma sustancial o potencia primitiva de actuar como una abstracción de lo único existente: la mónada. Tal como indican Rutherford 2008 (p. 142) y Adams 1994 (p. 274), tanto la materia prima como la forma sustancial son abstracciones que se hacen de la mónada y no tienen existencia alguna la una sin la otra en el sistema leibniziano, ni actual ni potencial.

El hecho de que la materia prima no sea más que una abstracción no implica que se relegue al plano ideal o al fenoménico. En efecto, a pesar de ser algo incompleto que se obtiene por un proceso de abstracción, Leibniz califica a la potencia primitiva pasiva de real con el fin de mostrar que con esa noción se refiere a un estado de la sustancia completa o mónada. En este sentido, advierte a De Volder que "se puede decir, en efecto, que la materia es real en cuanto que es en las sustancias simples la razón de lo que en los fenómenos observamos como pasivo" (GP II, 276; trad. OFC XVI, 1232-1233). Aquí la fuerza primitiva pasiva se diferencia de otras abstracciones como, por ejemplo, la extensión, la cual, como veremos en la tercera sección, no se abstrae de una sustancia simple, sino de una pluralidad de ellas, y no se refiere al estado de ninguna sustancia en particular, sino sólo a algún tipo de relación entre ellas.

\footnotetext{
${ }^{9}$ Cfr. supra n. 3.
} 
La materia prima leibniziana presenta una nota adicional que la aleja de nuevo de las posiciones tradicionales: es indivisible o inextensa, esto es, no contiene partes extra partes. Esta tesis puede encontrarse ya en la correspondencia con Arnauld, en la que Leibniz afirma:

Pero si se entendiese por el término materia algo que sea siempre esencial para la misma sustancia, se podría entender por ésta, en el sentido de algunos escolásticos, la potencia pasiva primitiva de una sustancia y, en ese sentido, la materia no sería en absoluto extensión ni sería divisible, aunque sí el principio de la divisibilidad o de lo que de ella corresponde a la sustancia. (A II, 2, 250-251; trad. OFC XIV, 134)

Como elemento esencial de las sustancias finitas, la materia prima sólo consiste en la capacidad de resistencia al cambio y, al igual que aquello de lo que se abstrae, no implica ni extensión ni divisibilidad. Ahora bien, Leibniz afirma que, a pesar de ser indivisible e inextensa, esta potencia primitiva pasiva es principio de la divisibilidad y de la extensión. ¿En qué sentido hay que entender esta tesis? Sobre este asunto, Adams afirma que "al parecer significa que es el principio del cual se sigue que la sustancia tenga un cuerpo divisible (y por ello extenso)" (Adams 1994, p. 348). Creo que el planteamiento leibniziano es aún más elemental: la materia prima como fuerza pasiva primitiva establece el carácter limitado o finito de la mónada y, en consecuencia, permite la pluralidad de sustancias. En efecto, la afirmación de que la materia prima es el principio de divisibilidad no significa sino que sirve como principio de limitación. Leibniz expone su posición a De Volder de la siguiente manera: "es necesario que haya en las cosas en cuanto limitadas un principio de limitación, como hay en cuanto agentes un principio de actividad" (GP II, 257; trad. OFC XVI, 1207). La materia prima, aunque inextensa en sí misma, permite la pluralidad de mónadas, a partir de las cuales podrá nacer la idea de extensión. De este modo, la materia prima no es ni infinitamente divisible (como será la extensión) ni tampoco es algo infinitamente dividido (como será la materia segunda - o como lo es la materia prima del periodo juvenil-), sino que es absolutamente indivisible (como la mónada de la cual no es más que una abstracción). Por lo tanto, con la noción de materia prima Leibniz se aleja no sólo del cartesianismo (en el cual no hay nada similar), sino también de la escolástica. Las series de características que he indicado en esta sección ayudarán a comprender en la siguiente cuál es la diferencia que la materia prima tiene con lo que Leibniz denomina "cuerpo orgánico". 


\section{Cuerpos orgánicos y fenómenos bien fundados}

El siguiente concepto que me interesa analizar es el de "cuerpo orgánico". Aun cuando esta noción tiene antecedentes en el periodo medio - sobre todo en la correspondencia con Arnauld-, recibe un análisis más acabado en los escritos maduros. De hecho, en torno a ella giran algunos de los principales debates que Leibniz mantuvo en los últimos años de su vida, como las polémicas con Des Bosses, Sturm o Stahl. Ahora bien, las notas del concepto leibniziano de cuerpo orgánico se manifiestan en su examen de la "materia segunda" (que también denomina "masa extensa" o incluso "cuerpo completo"). Estos términos no son equivalentes porque, aun cuando todo cuerpo orgánico es materia segunda, la relación inversa no es necesaria. En efecto, el cuerpo orgánico requiere algún principio en función del cual se organice el agregado de sustancias que llamamos materia segunda para poder constituir una sustancia corpórea. ${ }^{10} \mathrm{Mi}$ objetivo es determinar las características generales que Leibniz atribuye a la materia segunda, la cual, en la sustancia corpórea, recibirá el nombre de cuerpo orgánico. En virtud de ello, en esta sección trabajo en lo fundamental con la noción de materia segunda, que engloba a la de cuerpo orgánico, sin entrar en el debate acerca de la particularidad de este último (que implica ya tomar una posición respecto de cómo se entiende la sustancia corpórea). A continuación indico cuáles son las notas generales de la materia segunda y preciso cuáles son sus diferencias tanto respecto de la materia prima como de la extensión.

En primer lugar, Leibniz afirma que la materia segunda no es una sustancia, sino un agregado de sustancias. Subrayo esta característica porque, como veremos, es el cimiento a partir del cual se comprenden las características restantes de la materia segunda leibniziana:

La materia segunda o masa no es sustancia, sino sustancias; por lo tanto, no es el rebaño, sino el animal, ni es el cardumen, sino el pez lo que son una sustancia verdaderamente una. (GM III, 537)

La materia [segunda] son aquellas cosas mismas plurales en número y, por lo tanto, es agregado de aquellas cosas que contienen entelequias. (GP II, 195; trad. OFC XVI, 1125)

La materia (me refiero a la segunda), o una parte de la materia, existe de la misma manera que un rebaño o una casa, es decir: como ente por agregación. (GP II, 304; trad. OFC XIV, 171)

${ }^{10}$ Sobre este tema en particular, cfr. Duchesneau 2010 y Smith y Nachtomy 2011. 
La materia segunda (como, por ejemplo, el cuerpo orgánico) no es una sustancia, sino un ente de razón; es un agregado de muchas sustancias, como un estanque lleno de peces o como un rebaño de ovejas y, en consecuencia, no es más que un uno por accidente, esto es, un fenómeno. (GP III, 657)

Estos pasajes pertenecen a la correspondencia con Bernoulli, con De Volder, con Des Bosses y con Remond, respectivamente. Ahora bien, en el hecho de ser un agregado de sustancias yace la primera diferencia esencial entre la materia segunda y la materia prima: mientras que esta última no es una sustancia, sino sólo algo incompleto que requiere de la forma sustancial para constituir una sustancia, la primera es una pluralidad de sustancias completas o mónadas.

En segundo lugar, Leibniz afirma que, dado que no se trata de una sustancia, sino de una pluralidad de sustancias, la materia segunda no tiene una unidad por sí o sustancial, sino sólo una unidad por agregación o accidental y, por lo tanto, es un fenómeno. El carácter fenoménico de estos agregados es algo que Leibniz sostiene desde la correspondencia con Arnauld y mantiene hasta sus últimos escritos. A diferencia del filósofo alemán, quien no parece haber visto ningún inconveniente en esta tesis, intérpretes como Jolley 1986 (pp. 38-51), han planteado que caracterizar el cuerpo como agregado de sustancias y al mismo tiempo, como fenómeno, pretende reunir dos tesis irreconciliables; Jolley incluso afirma que en este punto radica la mayor inconsistencia de la metafísica leibniziana de los cuerpos. ${ }^{11}$ Más adelante me referiré a este problema y, en general, al debate en torno a las interpretaciones idealistas. Por el momento sólo señalo que la idea básica que permite establecer la equiparación entre unidad por agregación y fenómeno es, como Leibniz le comunica a De Volder, la siguiente:

Tal como yo entiendo la unidad [accidental], todas estas cosas se llaman con mayor corrección plurales y sólo constituyen unidad como agregados,

${ }^{11}$ Jolley 1986 plantea que, en los escritos maduros de Leibniz, hay dos teorías en competencia sobre la realidad del cuerpo: por un lado, la tesis que afirma que el cuerpo es fenómeno (reducible a percepciones coordinadas de mónadas); por otro, la idea de que el cuerpo es agregado de mónadas. En contra de la lectura fenomenalista de Furth 1967, quien defiende que Leibniz acepta el fenomenalismo, Jolley argumenta que el filósofo alemán no se decide por ninguna de las dos. En línea con lo que plantean Rutherford 1990a y 1990b y Adams 1994 (pp. 218-219), en mi trabajo sostengo que el debate en torno a la doble teoría sobre los cuerpos es, en verdad, una falsa disyuntiva. 
o sea, en la medida en que se comprehenden bajo un único acto del pensar. (GP II, 184; trad. OFC XVI, 1111)

Toda cosa que puede dividirse en pluralidades (que ya existen en acto) es agregado de esas pluralidades, y lo que es agregado de pluralidades no es uno sino en la mente y no tiene más realidad que la prestada por sus contenidos. (GP II, 267; trad. OFC XVI, 1221)

Estos pasajes muestran que, lejos de pensarlas como dos tesis irreconciliables, la posición de Leibniz es, como advierte Adams 1994 (p. 219), que los cuerpos son fenómenos porque son agregados. Con ello se quiere subrayar que la unidad de estos últimos no es algo que exista en sí mismo, sino sólo para quien la percibe. Por ejemplo, un rebaño o una pila de leños no tienen unidad sustancial aunque nos refiramos a ellos con un nombre, ya que, al margen de nuestra percepción, esto es, en la naturaleza, sólo hay ovejas y leños. Este ejemplo es sólo ilustrativo, ya que la idea leibniziana es que lo que existe independientemente de la percepción son mónadas. El caso de los leños quizá sea para Leibniz otro agregado de mónadas que sólo tienen una unidad accidental; las ovejas pertenecen al problemático caso de las sustancias corpóreas.

En tercer lugar, la materia segunda no se piensa como una composición de sustancias o mónadas, sino como un fenómeno resultante de una pluralidad de mónadas:

[L]a materia no se compone de unidades constitutivas, sino que resulta de ellas, pues la materia o masa extensa no es sino un fenómeno fundado en las cosas, como el arco iris o el parhelio, mientras que toda realidad lo es sólo de unidades. Así pues, los fenómenos siempre pueden dividirse en fenómenos menores, que podrían ser percibidos por animales más sutiles, pero nunca se llegará a fenómenos mínimos. Por el contrario, las unidades sustanciales no son partes de los fenómenos, sino fundamentos de ellos. (GP II, 268; trad. OFC XVI, 1222-1223)

Los cuerpos no son sino agregados, unidades por accidente o que se constituyen por denominación externa y, por ello, son fenómenos bien fundados. (GP VII, 344)

[L]a materia [segunda] no es sino un fenómeno, pero bien fundado, resultante de las mónadas. (GP III, 636; las cursivas son mías)

Estas afirmaciones se encuentran a lo largo de todo el periodo maduro, antes y después de la Monadologie; la última cita pertenece a una carta a Remond de febrero de 1715 . En relación con esta cuestión, Leib- 
niz es enfático al sostener que la materia segunda o cuerpo completo "no es una sustancia, sino un fenómeno resultante de las sustancias simples, que son las únicas que tienen unidad y absoluta realidad" (GP II, 275; trad. OFC XVI, 1231). Ahora bien, la materia segunda no es una composición de mónadas, sino sólo una pluralidad de ellas y de las cuales resulta como fenómeno lo que llamamos materia segunda. Por ello, Leibniz afirma que se trata de un fenómeno bien fundado, esto es, que se construye a partir de las cosas, unidades simples, reales e independientes de la percepción. Tal como indica Rutherford 2008 (p. 144), Leibniz marca aquí un límite a las lecturas fenomenalistas que sostienen que los cuerpos no tienen realidad independiente de los fenómenos $\mathrm{y}$, asimismo, que el fundamento de estos últimos se encuentra en la sola percepción armónica. En principio, un fenómeno bien fundado, tal como la materia segunda, es aquel que tiene como referencia no una sustancia, sino un agregado de sustancias. No obstante, sobre este tema hay un segundo grupo de afirmaciones de Leibniz que podrían hacer dudar respecto de cuál es el fundamento de estos fenómenos; se trata de una cuestión que abordaré luego de precisar otras características del cuerpo leibniziano.

En cuarto lugar, la materia segunda está dividida actualmente al infinito. En efecto, Leibniz sostiene: "en la masa corpórea, esto es, en la constitución de las cosas corpóreas, hay que desembocar en las unidades indivisibles como primeros constitutivos [...]. Los cuerpos son siempre divisibles; más aún, están divididos actualmente, pero no lo están sus constitutivos" (GP II, 267-268; trad. OFC XVI, 1222).

Por una parte, Leibniz advierte que la materia segunda está dividida actualmente; con ello se diferencia tanto de la materia prima, que es indivisible, como de la extensión que, como veremos, es infinitamente divisible. Esta tesis se deriva inmediatamente del hecho de que la materia segunda es un agregado de mónadas. Por otra parte, Leibniz añade que cualquier porción de materia segunda está dividida actualmente al infinito: "En realidad, la materia [segunda] no es algo continuo, sino algo discreto dividido actualmente hasta el infinito, aunque ninguna parte de espacio asignable está desprovista de materia" (GP II, 278; trad. OFC XVI, 1236).

En otras palabras, todo agregado es siempre un agregado de infinitas mónadas. ${ }^{12}$ En este punto confluyen las tesis sobre el infinito que se

${ }^{12}$ Sigo en este asunto la postura de Arthur 1998 (p. 25), según la cual el agregado de sustancias que hace al cuerpo orgánico debe ser necesariamente infinito. Leibniz explica esto a Des Bosses: "Me preguntas también por qué las mónadas son infinitas en acto. Respondo que para eso es suficiente su posibilidad, puesto que muestra la 
formulan durante el periodo de París y persisten aún en estos años. ${ }^{13}$ En efecto, Leibniz afirma que hay un infinito actual de mónadas en cada porción de materia segunda, pero ello no implica una cantidad infinita. Por el contrario, tal como se muestra en su correspondencia de la época, se mantiene firme en su posición acerca de la imposibilidad de las cantidades infinitas actuales:

[Y]o acepto una multitud infinita, pero tal multitud no hace un número o una totalidad; no es más que el hecho de que hay más términos que los que numéricamente pueden asignarse, exactamente como ocurre que se da la multitud o compuesto de todos los números sin que tal multitud sea un número o una totalidad. (GP III, 575)

Los argumentos contra el infinito en acto suponen que, una vez que se acepta que existe el número infinito, todos los infinitos son iguales. Pero es necesario saber que, en realidad, el agregado infinito ni es un todo, ni está dotado de cantidad, ni consta de número. En términos precisos, es necesario añadir que en lugar de hablar de número infinito habría que decir que hay muchas cosas que no se pueden expresar con número alguno. (GP II, 304; trad. OFC XIV, 173)

En la materia segunda hay una multitud infinita de sustancias que, empero, no es cuantificable, es decir, que es mayor a cualquier número que pueda asignársele. ${ }^{14}$ Tal como Leibniz plantea ya en su periodo parisino, la metafísica trata con elementos que escapan a la determinación numérica y geométrica. La multitud infinita de mónadas en toda materia segunda es una tesis capital para la constitución del sistema leibniziano, pues es la base para comprender por qué, a pesar de que el cuerpo es, en rigor, discreto (pues el agregado de mónadas no constituye un continuo), hay continuidad entre sus elementos (nota capital para poder extraer de allí la idea de extensión). En líneas generales, Leibniz defiende que, de la materia segunda, a pesar de ser discreta, puede abstraerse la extensión, que es continua e infinitamente divisible.

riqueza de la obra de Dios, pero lo exige además el orden de las cosas; de otra forma, los fenómenos no responderían a todas las percepciones asignables" (GP II, 460).

${ }^{13}$ Sobre la concepción leibniziana de las cantidades infinitas y su relación con la metafísica de los cuerpos, cfr. Fazio 2016a.

14 "En términos filosóficos yo no afirmo más las cantidades infinitamente pequeñas que las infinitamente grandes, es decir, ni más las infinitésimas que las infinitas. Para resumir, tanto unas como otras las considero ficciones de la mente, aptas para el cálculo, como lo son también las raíces imaginarias del álgebra" (GP II, 305; trad. OFC XIV, 172).

Diánoia, vol. LXIII, no. 80 (mayo de 2018). 
En quinto lugar, la materia segunda resulta de un agregado cuyas partes son determinadas y, por ello, es real: "En las cosas reales, o sea, en los cuerpos, las partes no son indefinidas (como lo son en el espacio, que es algo mental), sino que están actualmente asignadas de un modo definido" (GP II, 268; trad. OFC XVI, 1222).

Aunque el cuerpo no es sustancia, sino un fenómeno que resulta del agregado de sustancias, Leibniz afirma que se trata de una cosa real. Esta afirmación debe comprenderse en el contexto de la contraposición entre la materia segunda y los entes ideales como el espacio, el tiempo o el movimiento. Sin embargo, ¿por qué Leibniz afirma que el cuerpo, que es fenómeno, es una cosa real? Con esta expresión el filósofo alemán quiere enfatizar que el cuerpo es algo cuyas partes son determinadas y anteriores al todo. Por ello, se diferencia de las cosas en las que las partes son indeterminadas y posteriores al todo. ${ }^{15}$ En este sentido, por ejemplo, afirma a De Volder lo siguiente:

[E]l espacio, como el tiempo, no es algo sustancial, sino ideal, y consiste en la posibilidad u orden relacional, igualmente posible de los coexistentes. Así que en él no hay más división que aquella que la mente hace, ya que la parte es posterior al todo. Por el contrario, en las cosas reales las unidades son anteriores a la multiplicidad y no hay multiplicidad sino por las unidades. (GP II, 278; trad. OFC XVI, 1236)

La realidad de la materia segunda es, por así decirlo, derivada, pues se debe al hecho de que es un fenómeno bien fundado, esto es, una unidad mental que se funda en una pluralidad de unidades reales. En suma, aun cuando la materia segunda no es sustancia, es algo que descansa en una pluralidad de sustancias, mientras que elementos como el espacio o el tiempo no se refieren ni a una sustancia ni a una pluralidad de sustancias y, por eso, se califican de ideales.

Estas cinco características de la materia segunda, que Leibniz reconoce de manera explícita e insistente en sus escritos maduros, constituyen un límite infranqueable para las lecturas fenomenalistas que dominaron la escena desde principios hasta, al menos, finales del siglo xx, y que se encuentran en la actualidad en retirada. ${ }^{16} \mathrm{El}$ núcleo de esta propuesta

${ }^{15}$ En la filosofía leibniziana lo real siempre es discreto y determinado, mientras que lo continuo e indeterminado son notas propias de lo ideal. Sobre la importancia de esta idea para la comprensión de la metafísica leibniziana, cfr. Breger 1986.

${ }^{16} \mathrm{Si}$ bien con variaciones hermenéuticas, la tesis fenomenalista puede encontrarse en textos que van desde lecturas clásicas como la de Cassierer 1902 hasta intérpretes como Mahnke 1964, Martin 1964, Furth 1967, Broad 1975, MacDonald 
radica en afirmar que la materia segunda es un fenómeno fundado en la sola armonía de las percepciones entre las mónadas. De esta forma, la realidad del cuerpo se interpreta sólo en términos de regularidad y acuerdo intersubjetivo. Ahora bien, esta tesis tiene una base textual, pues Leibniz afirma en ocasiones que la concordancia de las percepciones es el fundamento del fenómeno. Uno de los pasajes más célebres y decisivos para justificar esta lectura pertenece a la correspondencia con De Volder, donde Leibniz sotiene:

Más aún, si se analiza esto con rigor, habrá que afirmar que nada hay en las cosas sino sustancias simples, y en ellas la percepción y el apetito; y que la materia y el movimiento, más que sustancias o cosas, son fenómenos de seres percipientes, cuya realidad reside en la armonía de los percipientes consigo mismos (según tiempos diversos) y con los demás percipientes. (GP II, 270; trad. OFC XVI, 1225)

Una afrimación similar se encuentra en el resumen que Leibniz ofrece de su metafísica de los cuerpos a Remond en 1714:

Todos los cuerpos y todo lo que les atribuimos no son sustancias, sino sólo fenómenos bien fundados, o el fundamento de apariencias, que son diferentes en los diferentes observadores, pero que tienen una relación $\mathrm{y}$ vienen de un mismo fundamento como las apariencias diferentes de una misma ciudad vista de distintos lugares [...]. No hay que concebir las mónadas como puntos que se mueven, se empujan o se tocan en el espacio real; es suficiente que los fenómenos hagan parecer que es así y esa apariencia contiene verdad en la medida en que estos fenómenos son fundados, es decir, concordantes. (GP III, 622-623)

Estos pasajes parecen contradecir los que cité antes y en los que Leibniz sostiene que la materia segunda es un fenómeno fundado en las cosas. Así, el interrogante que surge es si la metafísica leibniziana puede reducirse a una $u$ otra de estas dos tesis.

En línea con la crítica de Rutherford 1990a (p. 23), creo que la reducción fenomenalista, según la cual el fundamento de fenómenos tales como la materia segunda se encuentra en el solo acuerdo de percepciones, es problemática por su parcialidad. En efecto, esta interpretación

Ross 1984, Brown 1984, Jolley 1986 o Wilson 1989. En todas estas lecturas los cuerpos se conciben como fenómenos cuyo único fundamento radica en la consistencia de las percepciones de las mónadas, y nada más.

Diánoia, vol. LXIII, no. 80 (mayo de 2018). 
tiene dos fallas graves. En primer lugar, la base textual con la que trabaja es escasa y, a su vez, se ve obligada a desestimar muchos otros textos en los que Leibniz no razona en el mismo sentido. En segundo lugar, esta lectura no atiende las características que se reconocen a la materia segunda y que hasta aquí he expuesto, tales como que sea un agregado de sustancias, que esté dividido actualmente y que sus partes sean anteriores al todo. ${ }^{17}$ Dadas estas limitaciones hermenéuticas, es lícito preguntarse si es necesario optar por una de estas tesis. Sobre esta cuestión, pienso que Leibniz afirma ambas tesis justo porque son compatibles. En primer lugar, una prueba de ello es la carta a Remond antes citada, en la cual se afirma que el cuerpo es un agregado de mónadas y que es un fenómeno armónico, nota por la cual podemos estar seguros de que se trata de un fenómeno real y no imaginario. En ese pasaje es manifiesto que Leibniz cree que detrás de la percepción intersubjetiva concordante hay algo que subsiste, a saber, las cosas mismas. En segundo lugar, el propio Leibniz explica cómo se conjugan ambas tesis en la carta a Des Bosses del 29 de mayo de 1716: "Los agregados no son otra cosa que fenómenos, puesto que además de las mónadas que los constituyen, es sólo por la percepción que se añaden las otras cosas por el hecho mismo de que se perciben conjuntamente" (GP II, 517; trad. OFC XIV, 462; las cursivas son mías).

Las cualidades con las que percibimos la materia segunda se fundan en la sola percepción y no subsisten sin ella, pero las mónadas que forman el agregado subsisten al margen de tal acuerdo, aunque sin tales cualidades.

Por último, como sexta nota que caracteriza a la materia segunda, Leibniz afirma, en conexión con sus tesis dinámicas, que estos agregados de mónadas tienen a las fuerzas derivativas como cualidad esencial:

[E]n los fenómenos, esto es, en el agregado resultante, todo se explica ya de modo mecánico, entendiendo aquí que las masas actúan unas sobre otras de manera mutua, de manera que en estos fenómenos sólo es necesaria la consideración de las fuerzas derivativas, una vez que consta de dónde resultan éstas, a saber, los fenómenos de los agregados resultan de la realidad de las mónadas. (GP II, 250; trad. OFC XVI, 1199) ${ }^{18}$

${ }^{17}$ Tal como señala Hartz 2007 (p. 69), la subdivisión actual de la materia segunda es una tesis inexplicable bajo el presupuesto fenomenalista.

${ }^{18}$ En la correspondencia con Des Bosses se afirma en este mismo sentido: "así, de muchas mónadas resulta la materia segunda con las fuerzas derivativas, con acciones y pasiones, que no son más que entes por agregación y, por lo tanto, semimentales, como el arco iris y otros fenómenos bien fundados" (GP II, 306; trad. OFC XIV, 173). 
Este pasaje, además de retomar la equivalencia entre el agregado y el fenómeno, muestra que Leibniz reconoce las fuerzas derivativas como cualidad primera y fundamental de la materia segunda. Casos paradigmáticos de ésta son la fuerza motriz o, incluso, la inercia natural, las cuales no son ni movimiento ni reposo, sino precisamente la capacidad de causar el movimiento o resistirlo. Si dejamos de lado la tesis, que Leibniz nunca abandona, según la cual esas fuerzas son modificaciones de las fuerzas primitivas, cabe señalar en particular dos puntos. En primer lugar, Leibniz sostiene que las fuerzas derivativas son prioritarias y explican las otras cualidades de los cuerpos como la extensión o el movimiento. Al respecto vale la pena señalar que el principal aporte de la dinámica consiste en probar que estas fuerzas secundarias no consisten en una relación, como el movimiento o incluso la extensión, sino que pueden determinarse de modo absoluto en cada instante para cada cuerpo. En otras palabras, Leibniz juzga que la materia segunda es sujeto de fuerzas derivativas y que éste es el único predicado que puede definirse de manera categórica como perteneciente a un cuerpo completo o agregado de sustancias. En segundo lugar, estas fuerzas, a pesar de poder determinarse de modo absoluto, son momentáneas. En efecto, la materia segunda no es sino un agregado de sustancias que pasa de un estado a otro, pero cuyas fuerzas, empero, nacen y mueren a cada instante y es incapaz por sí mismo de retener la totalidad de sus modificaciones. Por ello, Leibniz se refiere a la materia segunda y al cuerpo orgánico como un río en flujo permanente; de hecho, el mayor desafío de las sustancias corpóreas nace de esa particularidad y radica en cómo brindar unidad a la corriente de fuerzas secundarias que constituyen la esencia de toda materia segunda.

Una vez que hemos precisado las características de la materia segunda, la distinción radical que separa esta noción de la de materia prima se vuelve evidente. De hecho, ambas se distinguen en cada una de sus notas. La materia prima es incompleta (una abstracción), indivisible y esencialmente una fuerza primitiva pasiva que se mantiene invariante respecto de todas las modificaciones de la mónada; la materia segunda es un agregado de sustancias completas o mónadas (un fenómeno), está dividida actualmente al infinito y, en esencia, es una fuerza derivativa pasiva que varía a cada instante. Ya que se comprenden estos dos conceptos puede analizarse cómo se construye a partir de ellos un tercer concepto vinculado con el cuerpo: la extensión. 


\section{Las mónadas, la masa extensa y la extensión}

Además de los dos conceptos que he expuesto hasta aquí, esto es, el de materia prima y la materia segunda, Leibniz distingue un tercero que se diferencia de ambos. En efecto, la metafísica leibniziana distingue entre la masa extensa (esto es, la materia segunda o cuerpo orgánico de la sustancia corpórea) y la extensión misma. Esta distinción es de gran importancia para la comprensión del sistema maduro y de ella depende la respuesta a un problema que preocupó a los intérpretes de Leibniz desde Wolff en adelante, a saber, cómo surge la extensión a partir de mónadas inextensas. ${ }^{19}$ En esta sección analizaré qué entiende Leibniz por extensión y cómo resulta ésta de los elementos básicos de su ontología; además, especificaré por qué no surge ni como cosa ni como fenómeno, sino como idea.

En el marco de las reflexiones sobre la noción de extensión, Leibniz introduce un nuevo elemento crucial, los entes ideales. Como veremos, la extensión y el espacio se vinculan de manera estrecha en la propuesta leibniziana. Ahora bien, respecto de este punto es imprescindible advertir que ni la mónada, ni la materia prima, ni el cuerpo orgánico o materia segunda se califican nunca de ideales. De hecho, aunque con sentidos muy dispares, Leibniz afirma que estos tres elementos son reales: la mónada es real en un sentido absoluto, la materia prima es real porque se refiere a un estado de la mónada y la materia segunda o fenómeno es real por ser algo que resulta de un agregado de mónadas. Una característica común de la materia prima y de la segunda es que encuentran cimiento en las mónadas. El caso de la extensión es distinto, porque consiste en una abstracción que se hace a partir de la materia segunda, pero que no hace referencia a la cualidad ni de una cosa real ni de un agregado de cosas reales, sino de un ente ideal: el espacio.

Con el fin de comprender cómo entiende Leibniz la extensión, conviene partir del concepto de materia segunda. A menudo, el filósofo alemán se refiere a ella simplemente como cuerpo, masa extensa o en forma directa como lo extenso. ¿Por qué Leibniz utiliza estos calificativos y cuál es su diferencia con la extensión? Para responder esto es necesario advertir tres características de la materia segunda leibniziana: la pluralidad, la coexistencia y la continuidad de sus partes constitutivas. Estas tres notas son las que permitirán abstraer y formar la noción de

\footnotetext{
${ }^{19}$ Esta pregunta constituyó un enigma para la tradición wolffiana en la medida en que se buscaba explicar cómo se compone el continuo de la extensión a partir de puntos inextensos. Sin embargo, de acuerdo con tales presupuestos, la respuesta es imposible (pues, a juicio de Leibniz, no hay composición del continuo).
}

Diánoia, vol. LXIII, no. 80 (mayo de 2018). 
extensión. Detengámonos de manera breve en ellas. Como imagen ilustrativa, Leibniz presenta a De Volder el siguiente caso para entender su posición:

Dado que todo cuerpo extenso, tal como realmente se encuentra en el mundo, es como un ejército de criaturas, como un rebaño o un conglomerado, lo mismo que un queso de gusanos, el nexo de cualquier parte con cualquiera otra del cuerpo no será más necesario que el que pueda darse entre las partes de un ejército; y de la misma manera que en un ejército unos soldados pueden sustituir a otros, así también en todo cuerpo extenso unas partes pueden sustituir a otras; ninguna parte tiene conexión necesaria con ninguna otra, de manera que, en la materia en general, si se elimina un elemento, es necesario que lo sustituya otro, exactamente lo mismo que ocurre cuando los soldados encerrados en un lugar estrecho (donde caben pocos), en cuanto sale uno es necesario que entre otro. (GP II, 193; trad. OFC XVI, 1123)

La materia segunda es un agregado de sustancias que, en sí misma, no tiene ninguna unidad, sino que se compone de unidades innumerables. ${ }^{20}$ Las notas de la pluralidad y de la coexistencia no son problemáticas pues, según la noción de Leibniz de la materia como agregado de sustancias, se sigue que en ella hay una multiplicidad de mónadas de manera simultánea. Respecto de la continuidad, la situación es algo más sutil. En principio, conviene recordar que Leibniz rechaza en forma explícita que un cuerpo sea algo continuo, puesto que, dado que es un agregado de mónadas, no es una unidad infinitamente divisible en la que las partes son posteriores al todo e indeterminadas, sino que es un conjunto discreto dividido actualmente en el que las partes están determinadas (aunque no numéricamente) y son anteriores al todo. El cuerpo es como un rebaño o un ejército; sus elementos no tienen conexión necesaria entre ellos y no forman una unidad real, sino sólo nominal. Sin embargo, Leibniz afirma que en el cuerpo hay continuidad, lo cual se comprende a partir de su propia tesis según la cual la multitud que forma un cuerpo es infinita en acto. En efecto, el infinito actual de mónadas es lo que garantiza que siempre unas mónadas sigan a otras sin que sea posible que haya brecha alguna entre ellas. Leibniz ilustra esta idea con la imagen de unos soldados que entran y

20 "En la masa de la extensión o, mejor, de los extensos o, como yo prefiero decir, en la multiplicidad de las cosas, afirmo que existe no una unidad, sino unidades innumerables" (GP II, 276; trad. OFC XVI, 1232).

Diánoia, vol. LXIII, no. 80 (mayo de 2018). 
salen de manera continua de un lugar estrecho. ${ }^{21}$ De este modo, las partes de la materia segunda tienen continuidad, pero no constituyen un continuo. Tras estas precisiones, pasemos al concepto de extensión.

El núcleo de la propuesta leibniziana radica en que la extensión no es más que algo que se abstrae de una pluralidad de cosas que tienen una relación entre sí, a saber, la continuidad y la coexistencia.

[L]a extensión es el abstracto de lo extenso, no es más sustancia que el número o la multiplicidad, y no expresa más que cierta difusión o repetición no sucesiva (como la duración), sino simultánea de una determinada naturaleza o, lo que es lo mismo, la multiplicidad de cosas de una misma naturaleza junto con alguna relación de orden de los existentes; una naturaleza, digo, que en efecto se dice extenderse y difundirse. Así pues, la noción de extensión es relativa, esto es, la extensión es extensión de algo, de la misma manera que decimos que la multiplicidad o la duración es multiplicidad de algo, duración de algo. (GP II, 269; trad. OFC XVI, 1223)

En términos rigurosos, la extensión es sólo algo modal; como el número y el tiempo, no es una cosa, pues es simplemente el modo abstracto como designamos la pluralidad posible continua de las cosas coexistentes, mientras que la materia son aquellas mismas cosas plurales en número y, por lo tanto, es agregado de aquellas cosas que contienen entelequias. (GP II, 195; trad. OFC XVI, 1125) 22

Tal como precisa Rutherford 2008 (pp. 155-156), Leibniz diferencia entre la extensión como un atributo abstraído de las apariencias fenoménicas del cuerpo y lo extenso, esto es, el cuerpo mismo, que identifica con una multitud de sustancias. De este modo, la extensión se piensa como algo que se abstrae, no de una única mónada como la materia prima, sino de un agregado de ellas, esto es, de la materia segunda. En particular, en la extensión se considera de forma abstracta una

${ }^{21}$ Sobre la diferencia entre la continuidad y lo continuo Leibniz se pronuncia, por ejemplo, en su correspondencia con Remond, donde afirma: "La continuidad no es algo ideal, sino que lo que allí hay de real se encuentra en el orden de la continuidad. En lo ideal o continuo el todo es anterior a las partes; como la unidad aritmética es anterior a las fracciones que la parten y que se pueden asignar arbitrariamente, las partes no son más que potenciales. En lo real lo simple es anterior al agregado, las partes son actuales, son anteriores al todo (GP III, 622623; las cursivas son mías).

22 "La extensión, si la distinguimos de los extensos, es algo abstracto, lo mismo que la duración o el número separado de las cosas, en el que la conexión de partes es igualmente necesaria que en la extensión" (GP II, 234; trad. OFC XVI, 1176; las cursivas son mías). 
relación particular entre las mónadas que forma el agregado: su orden simultáneo. Ahora bien, entre la materia segunda o masa extensa y la extensión misma —esto es, lo que Leibniz califica a veces de extensión matemática o directamente de cuerpo matemático- hay una diferencia crucial que Leibniz enfatiza en reiteradas ocasiones:

En la extensión matemática, con la que entendemos los posibles, no hay división actual ni más partes que las que hacemos con el pensamiento, ni hay primeros elementos, como tampoco en los números fraccionarios hay un mínimo que sea elemento de los restantes. Así pues, el número, la hora, la línea, el movimiento o el grado de velocidad y demás cantidades ideales o entes matemáticos no son en realidad agregados de partes, puesto que en ellos está totalmente indefinido el modo como uno quiera hacer la asignación de partes; y esto debe entenderse así de manera absoluta, porque no significan otra cosa que la mera posibilidad misma de asignar partes de cualquier manera. (GP II, 276; trad. OFC XVI, 1233; las cursivas son mías)

Como ya indiqué, la materia segunda o masa extensa es un agregado de mónadas cuyas partes, a pesar de ser una multitud infinita, están determinadas, esto es, son actuales y anteriores al todo. Por el contrario, en la extensión se produce la relación inversa: sus partes son indeterminadas, es decir, no son actuales, sino posibles y, por ello, pueden ser tantas como se quiera. En este caso el todo es anterior a las partes. Leibniz plantea que, por esta diferencia, la materia segunda es un fenómeno que puede calificarse de real en la medida en que es una unidad que, aun siendo accidental o por agregación y no sustancial, tiene fundamento en las cosas mismas, mientras que, por su parte, la extensión es una cantidad ideal.

En relación con la distinción que propongo entre el plano de lo fenoménico (el cuerpo) y el de lo ideal (la extensión), conviene hacer algunas aclaraciones, puesto que no es algo sobre lo que el propio Leibniz sea muy preciso. Por lo general, el término ideal no se predica de la extensión misma, sino del espacio, es decir, del sustrato que se piensa como sujeto de la extensión. Sin embargo, la referencia a la extensión como cantidad ideal puede entenderse si se atiende el tipo de abstracción peculiar que ella implica; en especial si se comprende la diferencia que esta abstracción tiene con la materia prima. Como hemos visto, la fuerza primitiva pasiva es la abstracción de un estado de la mónada y se califica de real por referirse a un estado de la sustancia; sin embargo, como se vio, tal fuerza no es ni sustancia ni un agregado de sustancias, sino algo incompleto. La extensión comparte con la materia 
prima el hecho de que es una abstracción y que no es ni sustancia ni agregado. No obstante, difiere en dos puntos radicales. En primer lugar, la extensión no se abstrae de una mónada, sino de un agregado de mónadas, es decir, de un fenómeno, algo cuya unidad no es sustancial, sino accidental, esto es, que no subsiste al margen de la percepción. En segundo lugar, en el caso de la extensión se abstrae una característica de la materia segunda particular y se considera como algo separado de las cosas mismas, o sea, una relación de posición entre sus elementos. Cabe indicar que el referente de esta abstracción no es un estado de la materia segunda, el cual, para Leibniz, sólo puede consistir en sus fuerzas derivativas, sino la relación de existencia simultánea de sus partes. En este sentido, una abstracción distinta que puede hacerse de la materia segunda y que no sería mera idealidad es, por ejemplo, la fuerza derivativa pasiva o inercia natural de un cuerpo. Leibniz juzga que tal noción también es algo abstracto, que no es ni sustancia ni fenómeno. A pesar de ello, tal potencia hace referencia a la capacidad del agregado de mónadas de resistir al cambio de estados. De este modo, pienso que conviene distinguir el tipo peculiar de abstracción que es la extensión. En ese sentido puede leerse la precisión sobre los tipos de abstracción que Leibniz presenta a Des Bosses: "Los predicados abstractos, que no son entes, son o bien esenciales (como los atributos o afecciones; los atributos son primitivos, las afecciones, derivadas) o accidentales, como las relaciones accidentales" (GP II, 472; trad. OFC XIV, 402).

En función de lo anterior puede comprenderse que la extensión es un predicado abstracto accidental. Sin embargo, con esto Leibniz no busca anular su validez o utilidad para las ciencias, sino sólo mostrar el carácter derivado de tal cualidad.

De este modo y a manera de conclusión, la metafísica leibniziana de los cuerpos propia del periodo maduro podría ordenarse en tres niveles: lo primero y elemental es la mónada con su materia prima y forma sustancial; lo segundo es lo que resulta de la mónada, esto es, la materia segunda o cuerpo orgánico -que se percibe y es un fenómeno bien fundado- y lo tercero es la relación que se abstrae del fenómeno, la extensión -que no se percibe, sino que sólo se piensa como idea-. Tal como señala Rutherford 2008 (p. 157), en este punto se torna fuerte la tentación de afirmar, junto con la tradición wolfianna, que no es difícil concebir cómo el cuerpo y el espacio se construyen a partir de mónadas. En este contexto, el intérprete recuerda el comienzo de la Estética trascendental como una muestra del vínculo estrecho que se concibe entre el cuerpo y la extensión: en efecto, frente al juicio "todos los cuerpos son extensos" (Ak A7/B11), Kant no duda en afirmar su carác- 
ter analítico a priori. Si se considera lo visto hasta aquí, es interesante observar cuál sería la posición de Leibniz sobre este tema en el marco de su ofensiva general contra la res extensa cartesiana que emprende en sus escritos maduros. En primer lugar, es obvio que la extensión no es algo que pertenezca al concepto de mónada ni a la materia prima. Sin embargo, ¿qué pasa con la materia segunda o agregado de mónadas? Leibniz reconoce que, en este caso, se trata de una masa extensa. Sin embargo, entiende que se trata de un agregado de mónadas en el que hay una repetición continua de una pluralidad de cosas que coexisten, pero no cree que ello implique la extensión matemática, pues la masa extensa no es infinitamente divisible ni se trata de una cantidad continua, notas propias de la extensión, sino que, por el contrario, esta infinitamente dividida y es discreta. Por ello, la extensión es un concepto incluido en la noción de espacio (ente ideal), pero no en la de cuerpo (agregado real). En suma, pienso que en este asunto radica el núcleo — problemático- de la posición de Leibniz frente a los filósofos modernos y, en particular, frente al cartesianismo imperante de fines del siglo XVII: escindir la noción de cuerpo de la de extensión.

\section{BIBLIOGRAFÍA}

Obras de Leibniz

A Sämtliche Schriften und Briefe, ed. Berlin-Brandenburgischen Akademie der Wissenschaften und der Wissenschaften in Göttingen, Akademie, Darmstadt/Leipzig/Berlín, 1923-.

FCS Nouvelles lettres et opuscules inédits de Leibniz, intr. A. Foucher de Careil, Librairie Philosophique de Ladrange, París, 1857.

GM Mathematische Schriften, 7 vols, ed. C.I Gerhardt, A. Asher, Berlín, 1848-1863.

GP Die philosophischen Schriften, ed. C.I. Gerhardt, 7 vols., Wiedeman Buchhandlung, Berlín, 1875-1890.

OFC II Obras filosóficas y científicas, vol. II, Metafísica, ed. A.L. González, Comares, Granada, 2010.

OFC XIV Obras filosóficas y científicas, vol. XIV, Correspondencia I, ed. J.A. Nicolás y M.R. Cubells, Comares, Granada, 2010.

OFC XVI Obras filosóficas y científicas vol. XVI, Correspondencia III, ed. B. Orio de Miguel, Comares, Granada, 2011.

Escritos filosóficos, ed. Ezequiel de Olaso, Mínimo Tránsito, Madrid, 2003.

Bibliografía secundaria

Adams, R., 1994, Leibniz. Determinist, Theist, Idealist, Oxford University Press, Oxford.

Diánoia, vol. LXIII, no. 80 (mayo de 2018). 
Arthur, R., 1998, "Infinite Aggregates and Phenomenal Wholes: Leibniz's Theory of Substance as a Solution to the Continuum Problem", The Leibniz Review, vol. 8, pp. 25-45.

Arthur, R. y P. Loptson, 2006, "Leibniz's Body Realism: Two Interpretations", The Leibniz Review, vol. 16, pp. 1-42.

Breger, H., 1986, "Das Kontinuum bei Leibniz", en A. Lamarra (comp.), L'infinito in Leibniz. Problemi e terminologia, Ateneo, Roma, pp. 53-67.

Broad, C.D., 1975, Leibniz. An Introduction, Cambridge University Press, Cambridge.

Brown, S., 1984, Leibniz, University of Minnesota Press, Mineápolis.

Cassirer, E., 1902, Leibniz' System in seinen wissenschaftlichen Grundlagen, Elwert, Marburgo.

Duchesneau, F., 2010, Leibniz. Le vivant et l'organisme, Vrin, París.

Fazio, R.E., 2016a, "La crítica de Leibniz a los números infinitos y su repercusión en la metafísica de los cuerpos", Theoria. An International Journal for Theory, History and Foundations of Science, vol. 31, no. 2, pp. 159-175.

_ 2016 b, "Leibniz y la reforma de la metafísica: un análisis de la definición de sustancia como fuerza primitiva activa", Revista Latinoamericana de Filosofía, vol. 42, no. 2, pp. 149-170.

— _ 2017, "Leibniz y la noción de sustancia corpórea en el período medio (1677-1695)", Anales del Seminario de Historia de la Filosofía, vol. 34, no. 1, pp. 105-125.

Furth, M., 1967, "Monadology", The Philosophical Review, vol. 76, no. 2, pp. 169-200.

Garber, D., 2009, Leibniz. Body, Substance, Monad, Oxford University Press, Oxford.

Hartz, G., 2007, Leibniz's Final System. Monads, Matter and Animals, Routledge, Londres/Nueva York.

— 2008 , "Why Corporeal Substances Keep Popping Up in Leibniz's Later Philosophy", British Journal for the History of Philosophy, vol. 6, no. 2, pp. 193-207.

Jolley, N., 1986, "Leibniz and Phenomenalism", Studia Leibnitiana, vol. 18, pp. $38-51$.

Kant, I., 1968, Kants Werke. Akademie Text Ausgabe, Walter de Gruyer, Berlín [citado como Ak].

MacDonald Ross, G., 1984, "Leibniz's Phenomenalism and the Construction of Matter", Studia Leibnitiana, vol. 13, pp. 26-36.

Mahnke, D., 1946, Leibnizens Synthese von Universalmathematik und Individualmetaphysik, F. Frommann, Stuttgart.

Martin, G., 1964, Leibniz. Logic and Metaphysics, trad. K.J. Northcott y P.G. Lucas, Manchester University Press, Manchester.

Phemister, P., 2005, Leibniz and the Natural World. Activity, Passivity and the Corporeal Substance, Springer, Dordrecht.

Rutherford, D., 1990a, "Phenomenalism and the Reality of Body in Leibniz's Later Philosophy", Studia Leibnitiana, vol. 12, pp. 11-28. 
Rutherford, D., 1990b, "Leibniz's Analysis of Multitude and Phenomena into Unities and Reality", Journal of the History of Philosophy, vol. 28, pp. 525552.

, 2008, "Leibniz as Idealist", Oxford Studies in Early Modern Philosophy, vol. 4, pp. 141-190.

Smith, J. y O. Nachtomy, (comps.), 2011, Machines of Nature and Corporeal Substances in Leibniz, Springer, Dordrecht.

Wilson, C., 1989, Leibniz' Metaphysics. A Historical and Comparative Study, Manchester University Press, Manchester.

Recibido el 20 de junio 2016; aceptado el 25 de mayo de 2017.

Diánoia, vol. LXIII, no. 80 (mayo de 2018). 